\title{
Analysis of Spatial Epidemic Characteristics of Liver Cancer in Small Geographical Area
}

\author{
Jing $Q u^{1,3}$, Haiyun Liu ${ }^{2}$, Jian $X u e^{2}$, Jianjun $X u^{2}$, Maobo Wang ${ }^{2, *}$, Fuzhong Xue ${ }^{3, *}$ \\ ${ }^{1}$ Yantai Longkou County Center for Disease Control and Prevention, Yantai, China \\ ${ }^{2}$ Yantai Center for Disease Control and Prevention, Yantai, China \\ ${ }^{3}$ Department of Epidemiology and Health Statistics, School of Public Health, Shandong University, Jinan, China \\ Email address: \\ 362089762@qq.com (Jing Qu), email2 ytwmb@sina.com (Maobo Wang), email3 xuefzh@sdu.edu.cn (Fuzhong Xue) \\ ${ }^{*}$ Corresponding author
}

\section{To cite this article:}

Jing Qu, Haiyun Liu, Jian Xue, Jianjun Xu, Maobo Wang, Fuzhong Xue. Analysis of Spatial Epidemic Characteristics of Liver Cancer in Small Geographical Area. Journal of Cancer Treatment and Research. Vol. 8, No. 1, 2020, pp. 10-17. doi: 10.11648/j.jctr.20200801.12

Received: December 17, 2019; Accepted: January 3, 2020; Published: January 13, 2020

\begin{abstract}
Background: At present, the spatial studies of liver cancer are too large for observation, or lack systematic spatial epidemics and statistical analyses, so it is difficult to put forward more targeted regional prevention and control measures. This study takes the natural village of Longkou City, Shandong as a small regional research unit, to systematically analyze the spatial structure and spatial heterogeneity of liver cancer in the small region (village as a unit), and provide a scientific basis for the development of regional prevention and control measures for more targeted liver cancer and its related diseases. Methods: Based on the database of the integrated management information system for chronic diseases and death monitoring in Shandong Center for Disease Control and Prevention, the spatial epidemiology and spatial statistics methods were combined in the framework of geographic information system (GIS). Results: The results of spatial distribution showed that there was obvious spatial heterogeneity on liver cancer in Longkou City, and there were many high incidence and high death areas scattered in the distribution. The spatial scanning and space-time scanning results of liver cancer showed that there were relatively fixed high incidence and high death villages in the small area of Longkou City. Conclusion: This study suggests that the liver cancer in Longkou City has a local geographic risk factor, which may have obvious high incidence area, and time factors have a certain influence on the space area of liver cancer. It is necessary to further carry out the geographical weight regression and risk factors analysis, and expand the study model.
\end{abstract}

Keywords: Small Geographical Area, Liver Cancer, Spatial Epidemiology

\section{Introduction}

With the application of GIS in the research of chronic diseases, the studies on the spatial distribution of liver cancer have been published. Surveillance of geographical differences in disease prevalence and understanding of root causes are important starting points for cancer epidemiology, [1]. Studies from Li Bing et al. have shown that in China, the incidence and mortality of liver cancer also have obvious regional distribution characteristics, [2, 3]. In the early study, Chen Wanqing et al. pointed out that Jiangsu, Guangxi, Fujian and Heilongjiang were the provinces with higher liver cancer death rate, and Yunnan, Guizhou, Beijing, Tianjin and other provinces and cities were the provinces and cities with relatively lower liver cancer rate, [4]. Weng Min et al. took street as the research unit, using geographically weighted regression to quantitatively analyze the spatial relationship between liver cancer incidence and social deprivation [5]. However, these studies, either are too large for observation in scale, or lack systematic spatial epidemics and statistical analysis, so it is still difficult to put forward more targeted regional prevention and control measures. Therefore, this study takes the natural village of Longkou City in Shandong as a small regional research unit. Based on the database of the information system for the chronic disease and death monitoring in the Center for Disease Control and Prevention in Shandong, and in the framework of GIS, the study combines the spatial epidemiology and the spatial statistics method to analyze the spatial structure and spatial 
heterogeneity of hepatocellular carcinoma (HCC) in a small region (village as a unit), and provide a scientific basis for the development of regional prevention and control measures for more targeted liver cancer and related diseases.

\section{Information and Methods}

\subsection{Source of Information}

The population data of 647 natural villages in Longkou City derives from the data of the 2010 census in Longkou City, and the data of liver cancer in $2012 \sim 2016$ comes from the "chronic disease monitoring information management system" and "the information management system for the death cause monitoring" from the Longkou City Center for Disease Control and prevention.

\subsection{Spatial Statistical Analysis Method}

\subsubsection{Construct GIS Map, Plot Cumulative Rate Map and Excess Hazard Ratio Map}

The liver cancer data collected in Longkou City, Shandong, in $2012 \sim 2016$, is associated with the vector map in the unit of village to construct a corresponding GIS map, where different levels of the disease can be visually expressed in different colors, and thus to draw the cumulative rate map and the map of the excess risk ratio. Excess hazard ratios (EHR) refer to the ratios of cumulative incidence or cumulative mortality of a natural village to the cumulative or cumulative mortality rate in the whole Longkou region [6].

\subsubsection{The Establishment of Space Weight Matrix}

The establishment of the spatial weight matrix is the basis and prerequisite for spatial exploration analysis. It represents the spatial position information in the form of data value and forms the spatial weight matrix of $n \times n$, where the spatial position information is expressed as $w_{n \times n}$ and the value lying in the $i$ th row and $j$ th line represents the spatial weight [7] between the $i$ th and the $j$ th regions.

\subsubsection{Spatial Autocorrelation Analysis}

In this study, the Moran's $I$ statistics were used for global spatial autocorrelation and local spatial autocorrelation (Local Indictors of Spatial Autocorrelation, LISA) to analyze the correlation and direction of the overall and local levels of liver cancer in Longkou City during the 2012 2016 period.

The expression of the global Moran's I statistic is [8]:

$$
I=\frac{n}{\sum_{i=1}^{n} \sum_{j=1}^{n} w_{i j}} \cdot \frac{\sum_{i=1}^{n} \sum_{j=1}^{n} w_{i j}\left(x_{i}-\bar{x}\right)\left(x_{j}-\bar{x}\right)}{\sum_{i=1}^{n}\left(x_{i}-\bar{x}\right)^{2}}
$$

Among which, $n$ represents the number of the space units (the number of the villages), $x_{i}$ represents the incidence density of the $i$ th liver cancer unit, $\bar{x}$ indicates the incidence density of the liver cancer in the whole study area and $w_{i j}$ indicates the weight matrix. The range of global Moran's $I$ statistics is $(-1,1)$; when $I=0$, it indicates no spatial correlation; when $I>0$, it represents positive spatial correlation; and when $I<0$, it represents negative correlation of space; $|I|$ can reflect the close degree of spatial autocorrelation.

The expression of the local Moran's I statistics is [9]:

$$
I_{i}=\frac{\left(x_{i}-\bar{x}\right)}{\sum_{j=1, j \neq i}^{n}\left(x_{j}-\bar{x}\right)^{2} /(n-1)-\bar{x}^{2}} \sum_{j=1, j \neq i}^{n} w_{i j}\left(x_{j}-\bar{x}\right)
$$

Among which, $n$ stands for the number of space units in the whole research area, $w_{i j}$ is the spatial weight matrix, and the other parameters are the same as those above.

\subsubsection{Space Scanning Statistics}

In this study, we use the Poisson distribution model in space scanning statistics and space-time scanning statistics to study the spatial aggregation, time-place clustering, the exact location and risk size $[10,11]$ of liver cancer in Longkou City, and the calculation formula is:

$$
L L R=\log \left(\frac{n}{E(n)}\right)^{n}\left(\frac{N-n}{N-E(n)}\right)^{N-n} I()
$$

Among which, $n$ represents the number of actual incidence or death cases within the scanning window, $N$ is the total number of actual cases occurring in the whole study area, $E$ (n) is the expected incidence in the scanning window after the corresponding covariate is corrected under the condition of zero hypothesis, and $I O$ is exponential function. If the actual number of the incidence in the window is lower than the expected number, then $I()=0$; otherwise, $I()=1$. Each scan window corresponds to a $L L R$ value, and the largest $L L R$ corresponding region is the first (most likely) clustering region [12].

\subsubsection{Statistical Analysis Software}

Office Excel 2007 software is used for data sorting. ArcGIS 10.2 software is used to draw spatial distribution map. GeoDa 1.8 software is used to analyze global and local spatial autocorrelation. SatScan software is used to realize spatial scanning.

\section{Results}

\subsection{Spatial Distribution of Liver Cancer in Longkou City}

The cumulative incidence and cumulative mortality rate maps of liver cancer in Longkou City from 2012 to 2016 are shown in Figure 1 and Figure 2, respectively. The figures show: there is spatial heterogeneity in the cumulative incidence of liver cancer, multiple high incidence areas can be found in the map, and these high incidence areas are punctuated and distributed, with obvious variance even in the adjacent areas. The cumulative mortality of liver cancer also bears spatial heterogeneity, and the cumulative mortality rates in adjacent areas are also significantly different. The regions, respectively with highest cumulative incidence and highest cumulative mortality of liver cancer were different, and the mortality regions changed less than incidence regions. 


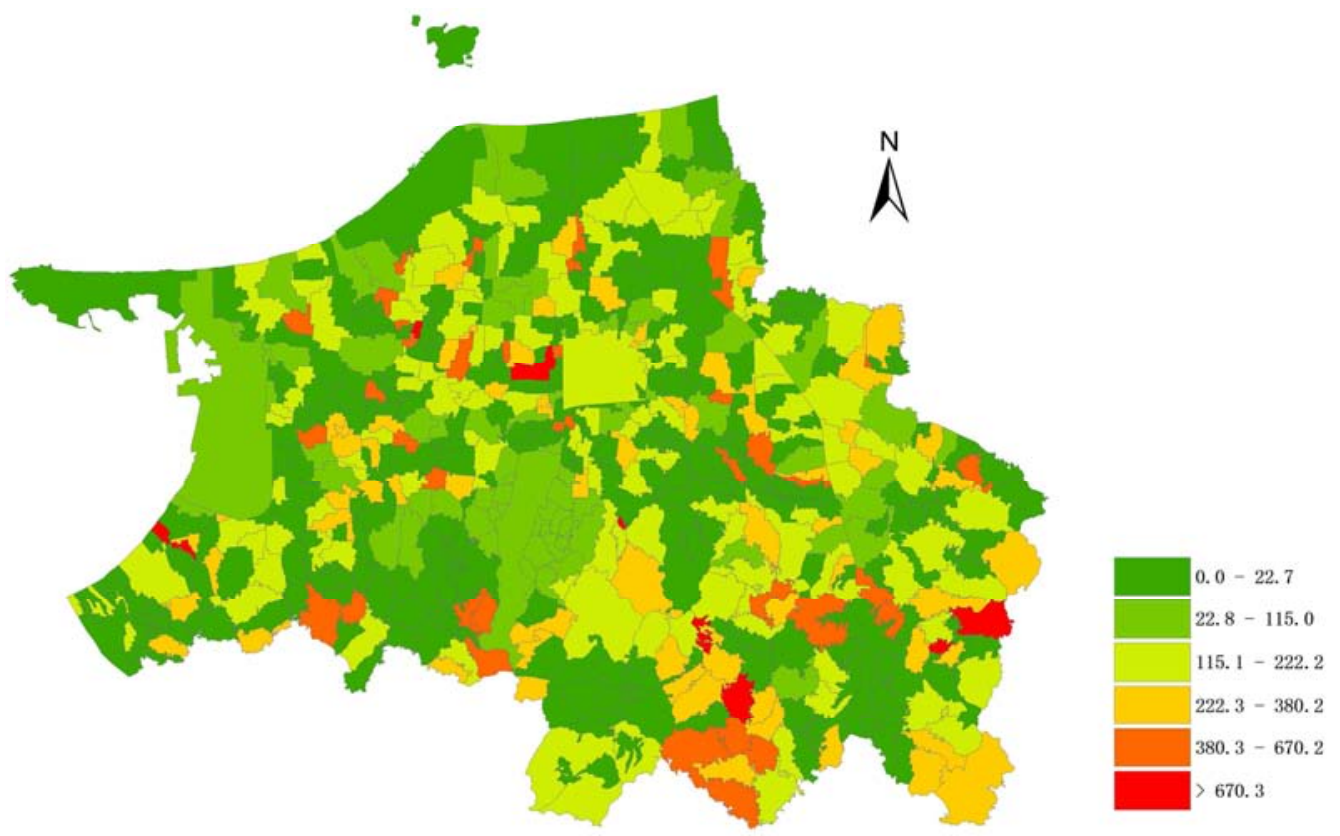

Figure 1. Map of cumulative incidence rate of liver cancer in Longkou City in 2012 2016 (/100000).

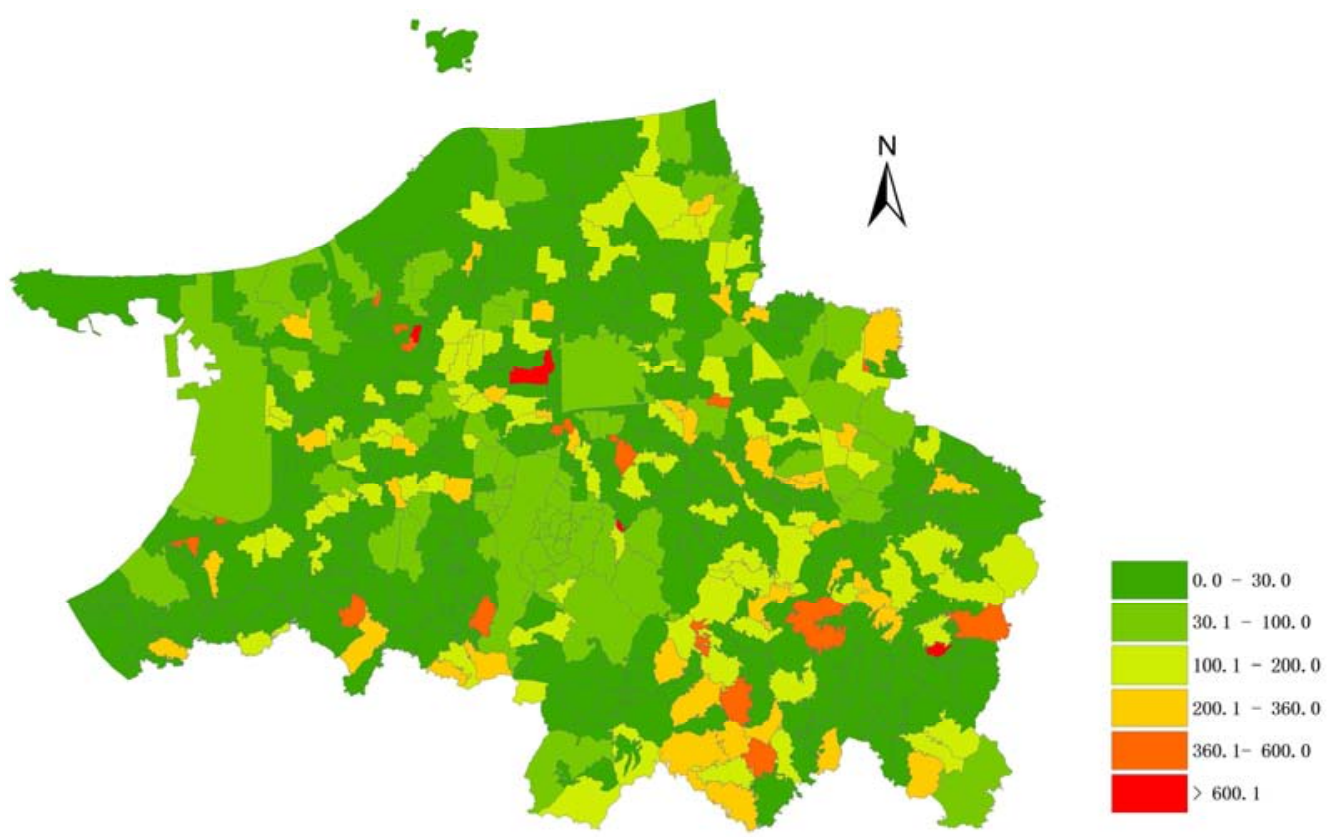

Figure 2. Map of cumulative mortality rate of liver cancer in Longkou City in 2012 2016 (/100000).

\subsection{Spatial Distribution of Excess Risk Ratio (EHR) of Hepatocellular Carcinoma in Longkou City}

According to the ratio of cumulative incidence of liver cancer in village level to that of the whole Longkou City, a map is drawn. The results are as shown in Figure 3. It is shown that, in the spatial distribution of liver cancer, the HER of cumulative incidence all bear spatial heterogeneity, and many high EHR areas (villages of EHR >6) can be found in the map, besides these high EHR regions are also punctuated in distribution and there are also significant differences in the EHRs of the adjacent areas; and in the spatial distribution grade map, the high EHR areas also vary one from another. Based on the ratio of the cumulative death rate of the village level to the total mortality rate in Longkou City, a map is drawn. The results are as shown in Figure 4. The map shows that, in the spatial distribution of liver cancer, the EHR of cumulative mortality has spatial heterogeneity, and there are many high EHR areas (villages of EHR>11) in the map, besides, these high EHR regions are still punctuated through the map and the distribution of HER in the adjacent areas still has significant differences; while in the spatial distribution grade, the high HER areas similarly vary one from another. 


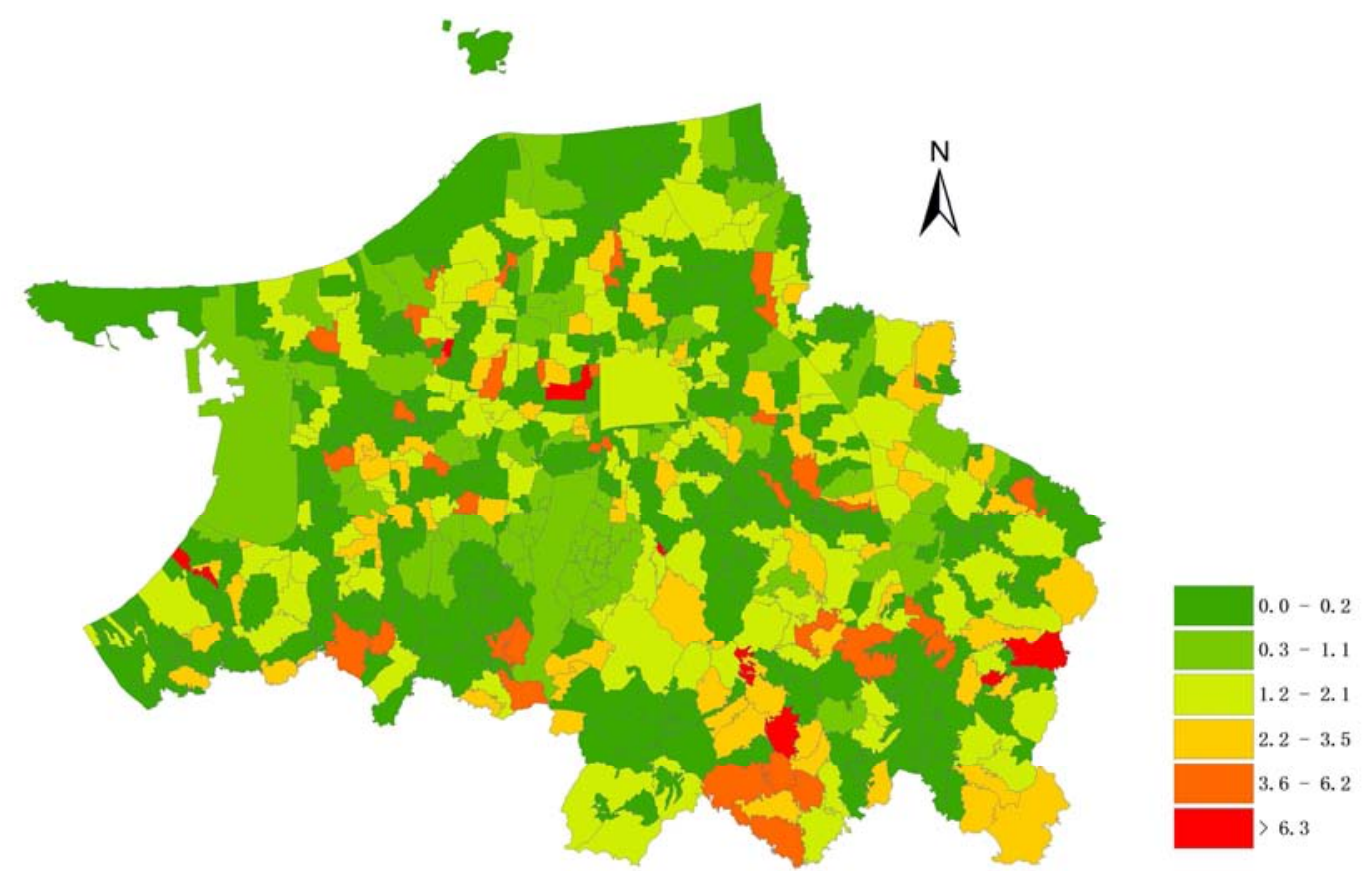

Figure 3. EHR grade map of liver cancer incidence in Longkou City in 2012 2016.

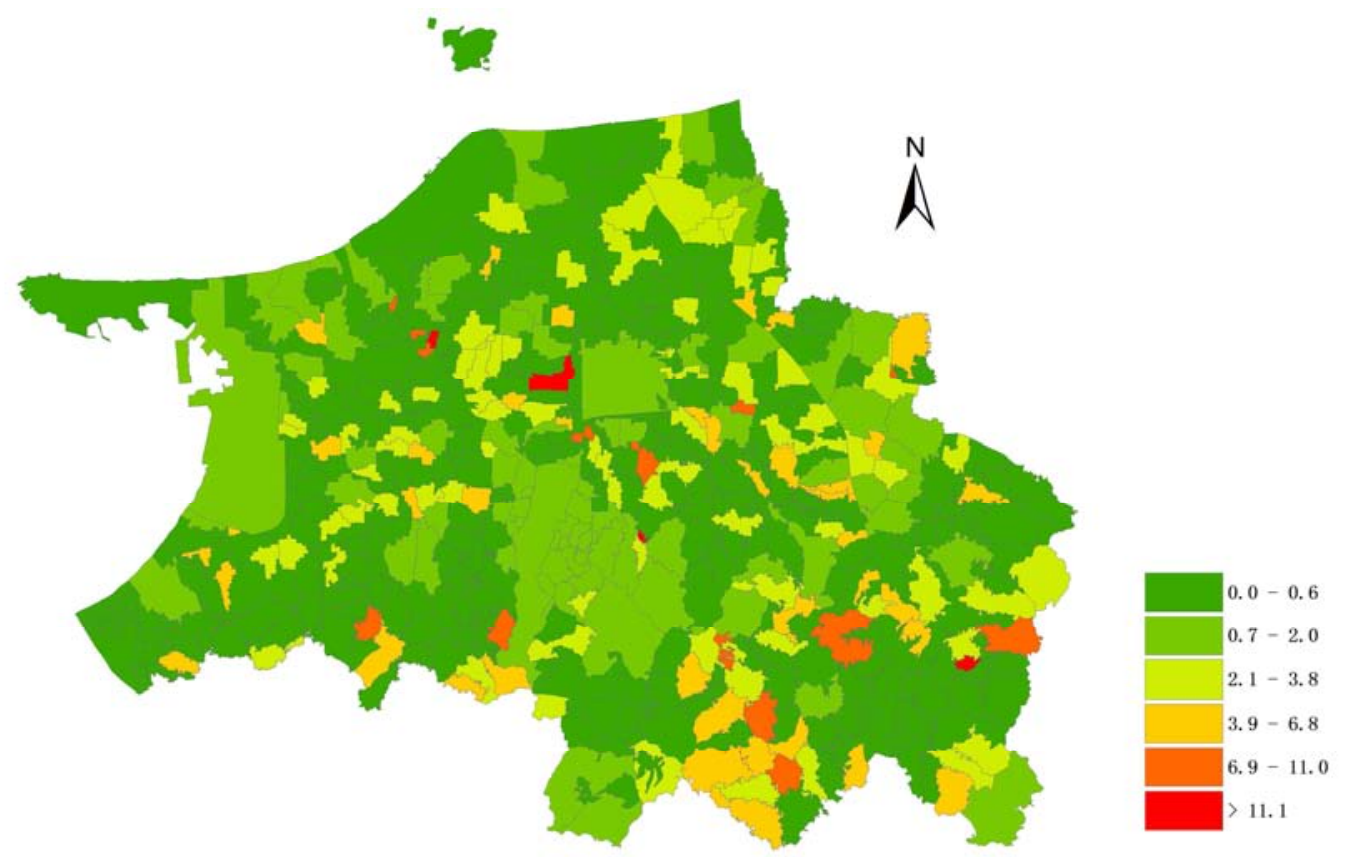

Figure 4. EHR grade map of liver cancer death in Longkou City in $2012 \sim 2016$.

\subsection{Spatial Autocorrelation Analysis of Liver Cancer in Longkou City}

First, the map of Longkou City village level is weighted, the spatial weighting matrix is established, and then the spatial autocorrelation analysis is carried out. The results are shown in Table 1 and Figure 5, Figure 6 and Figure 7, among which, Table 1 and Figure 5 (left: incidence and right: mortality) are the results of Moran's I global spatial autocorrelation analysis, and Figure 6 and Figure 7 are the results of LISA analysis.

From the Moran's I global spatial autocorrelation analysis, the Moran's I value (Mulan index), E (I) value (global correlation statistics), $\operatorname{Var}(I)$ value (global correlation statistic standard deviation) and $P$ value are obtained. The results show that the cumulative incidence of liver cancer has spatial autocorrelation (Moran's $I=0.0486, P=0.031$ ), indicating the accumulation of liver cancer in Longkou City takes on positive spatial correlation, and there may exist high incidence areas. The cumulative mortality of liver cancer is not statistically significant (Moran's $I=0.0208, P=0.177$ ), and there is no spatial correlation. 
Table 1. Global spatial autocorrelation of liver cancer Moran's I in Longkou City in 2012 2016.

\begin{tabular}{lllll}
\hline Category & Moran's $\boldsymbol{I}$ & $\boldsymbol{E}(\boldsymbol{I})$ & $\boldsymbol{V a r}(\boldsymbol{I})$ & \\
\hline Cumulative incidence rate & 0.0486 & -0.0015 & 2.0927 & 0.031 \\
Cumulative mortality rate & 0.0208 & -0.0015 & 0.9463 & 0.177 \\
\hline
\end{tabular}

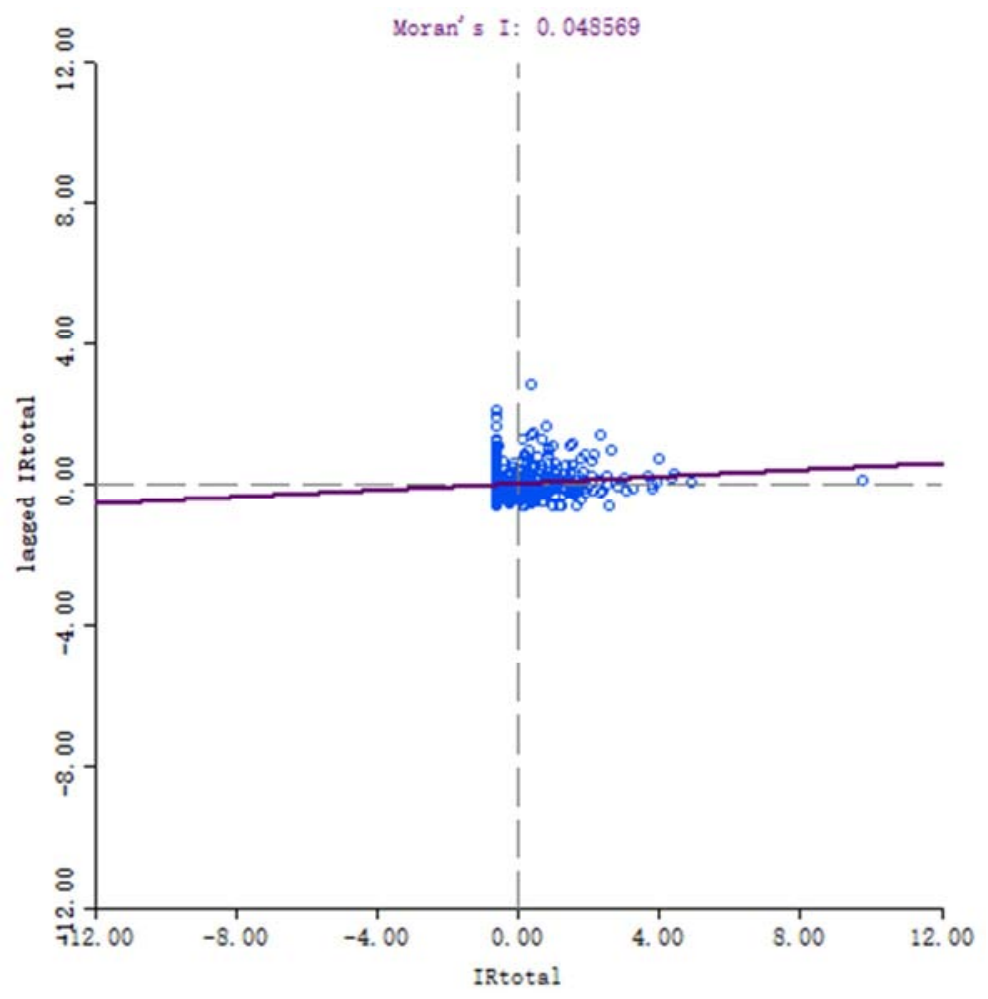

(a)

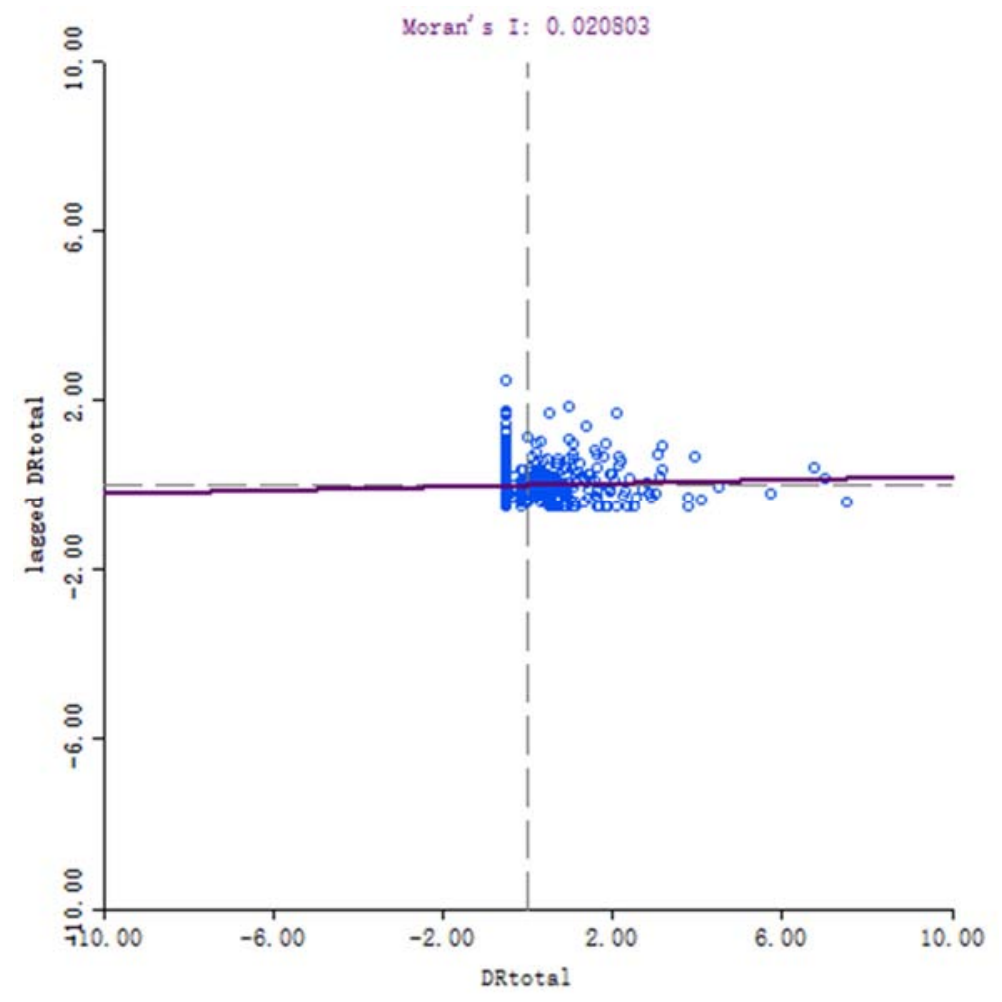

(b)

Figure 5. Spatial autocorrelation Moran's I scatter plot of liver cancer in Longkou City in $2012 \sim 2016$. 
The LISA analysis of liver cancer shows that, as for cumulative incidence, the LISA of 65 villages is statistically significant, including 17 high-high aggregation areas, 12 low-low aggregation areas, 19 low-high aggregation areas, and 17 high-low aggregation areas; as for cumulative mortality, the LISA of 65 villages is with statistical significance, including 12 high-high aggregation areas, 6 low-low aggregation areas, 25 low-high aggregation areas and 22 high-low aggregation areas. No matter for incidence or mortality, the high-high aggregation areas of liver cancer are mainly distributed in the northeast, low-low aggregation areas, low-high aggregation areas and high-low aggregation areas are scattered in distribution, and mainly concentrated in inland and less in coastal areas.
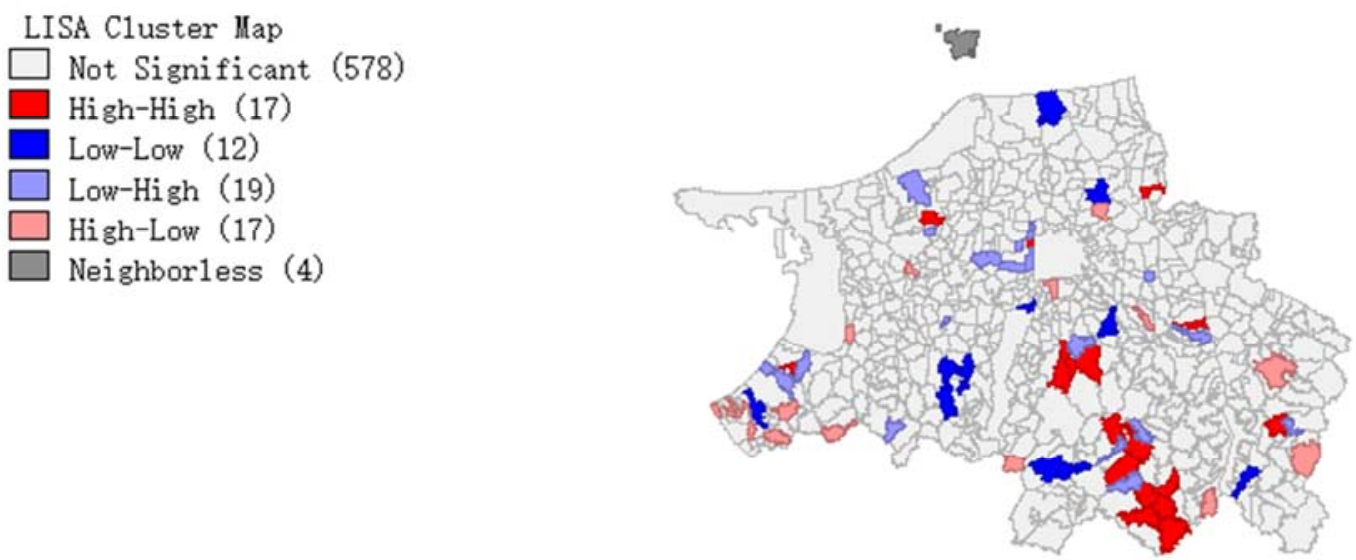

Figure 6. Spatial autocorrelation LISA map of cumulative incidence of liver cancer in Longkou City in $2012 \sim 2016$.
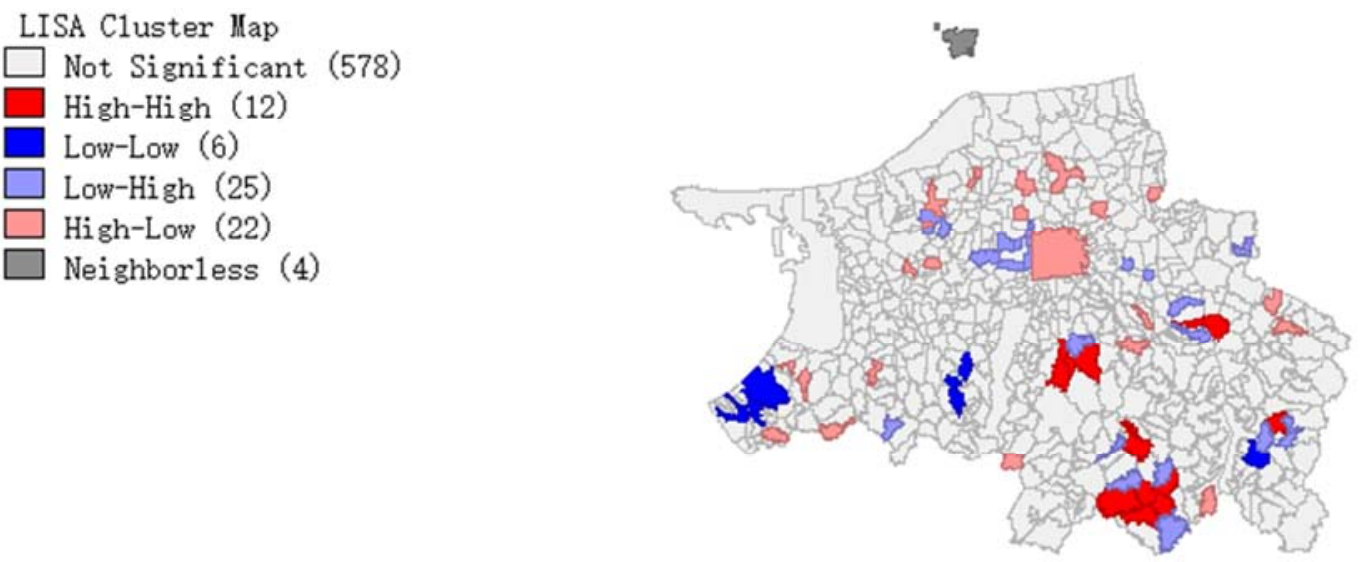

Figure 7. Spatial autocorrelation LISA map of cumulative mortality of hepatocellular carcinoma in Longkou City in $2012 \sim 2016$.

\subsection{Spatial Scanning and Space-time Scanning of Liver Cancer in Longkou City}

The incidence and death conditions of liver cancer in Longkou City are scanned with village as unit. The results are shown in Table 2, Figure 8 and Figure 9. The total cumulative incidence rate of liver cancer include 1 most likely aggregation area (the first aggregation area) and 1 sub possible aggregated area (the second aggregation area), of which, the first gathering area is mainly located in the northeast of Longkou City and contains 204 villages, with the relative risk
1.88 and the $\log$ likelihood ratio 30.86 , while the second gathering area is mainly located in the west coast of Longkou City, including 151 villages, with the relative risk 1.43 and the logarithmic likelihood ratio 13.01. The total cumulative death rate of the liver cancer covers only 1 most likely aggregation area (the first gathering area). The area is also located in the northeast of Longkou City, but the coverage area is much less than that of the incidence of 67 villages. Otherwise, the relative risk is 3.06 , and the logarithmic likelihood ratio is 21.79 .

Table 2. Spatial scanning analysis of liver cancer in Longkou City in $2012 \sim 2016$.

\begin{tabular}{|c|c|c|c|c|c|c|c|c|}
\hline Category & Aggregation type & Number & Radius & Observation number & Expectation number & $R R$ & $L L R$ & $P$ value \\
\hline \multirow{2}{*}{ Cumulative IR } & First aggregation area & 204 & 11.51 & 216 & 125.05 & 1.88 & 30.86 & $<0.0001$ \\
\hline & Second aggregation area & 151 & 7.51 & 276 & 206.26 & 1.43 & 13.01 & 0.0013 \\
\hline Cumulative MR & First aggregation area & 67 & 7.34 & 51 & 17.56 & 3.06 & 21.79 & $<0.0001$ \\
\hline
\end{tabular}




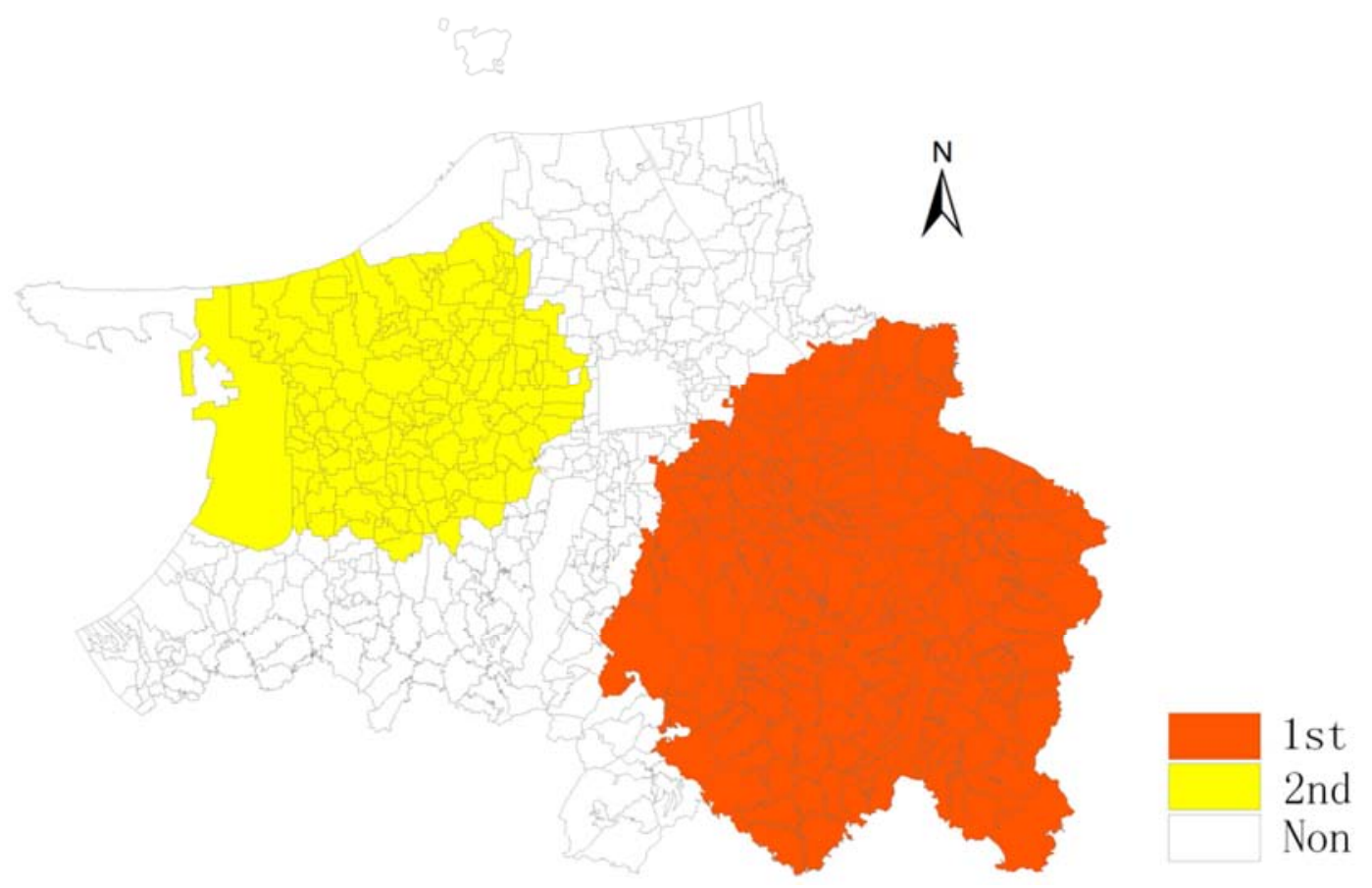

Figure 8. Spatial scanning map of cumulative incidence of liver cancer in Longkou City in 2012 2016.

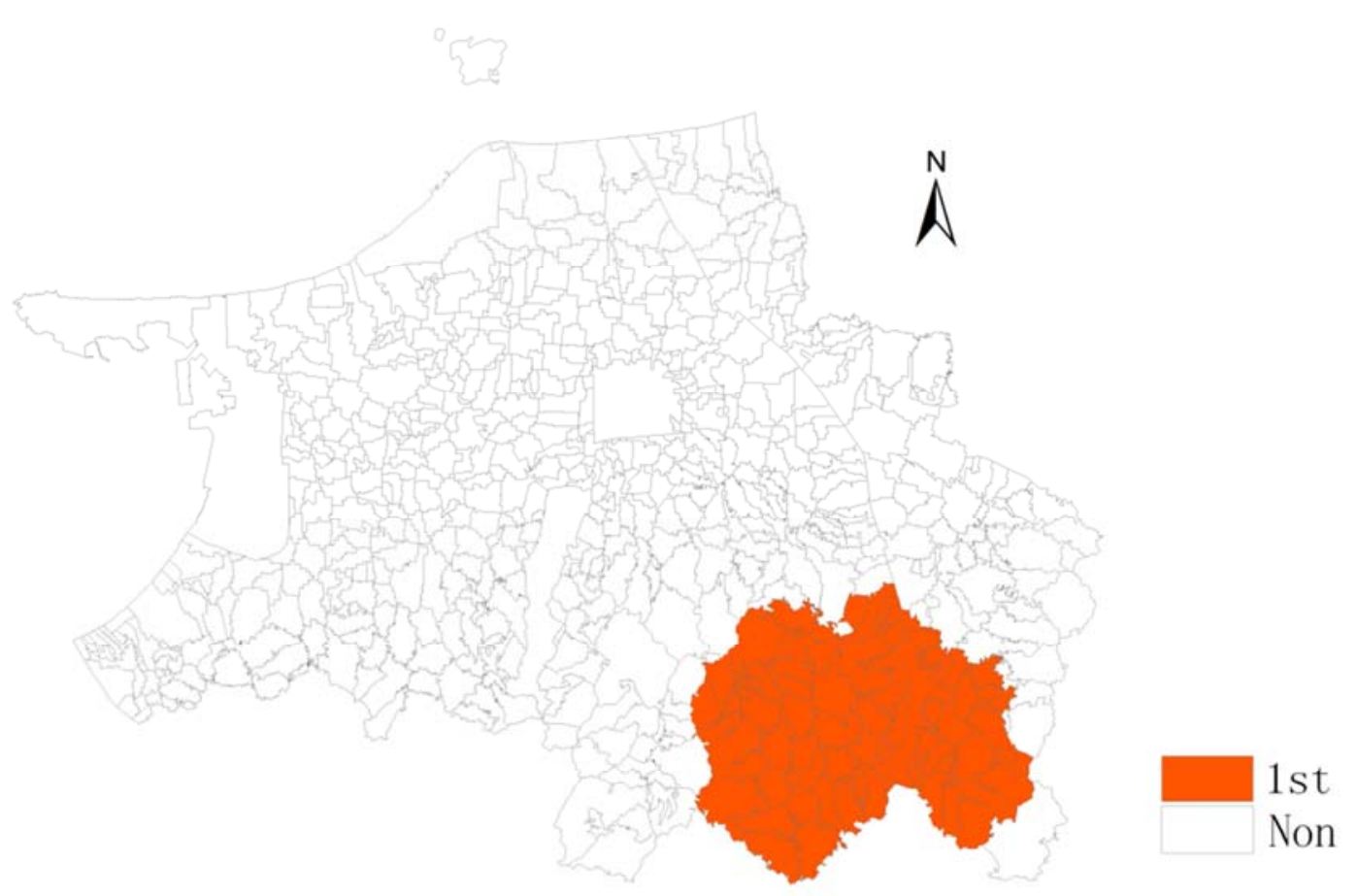

Figure 9. Spatial scaning map of cumulative mortality of hepatocellular carcinoma in Longkou City in 2012 2016.

The time and space scanning of the incidence and death of liver cancer in Longkou City was scanned by the unit of village to analyze the time changes of liver cancer in Longkou City. As shown in Table 3, it can be seen that the results of spatio-temporal scanning and simple spatial scanning are different, indicating that the time factors have certain impact on both high incidence space areas and high mortality space areas of the liver cancer.

Table 3. Spatial and temporal scanning results of liver cancer in Longkou City in 2012 2016.

\begin{tabular}{|c|c|c|c|c|c|c|c|c|c|}
\hline Category & Aggregation type & Number & Time slot & Radius & Observation number & Expectation number & $R R$ & $L L R$ & $P$ value \\
\hline Cumulative IR & First aggregation area & 90 & $14-15$ & 8.45 & 58 & 20.24 & 2.96 & 23.89 & $<0.0001$ \\
\hline \multirow{2}{*}{ Cumulative MR } & First aggregation area & 87 & 12 & 5.06 & 133 & 66.52 & 2.24 & 29.37 & $<0.0001$ \\
\hline & Second aggregation area & 206 & $14-15$ & 19.03 & 66 & 26.93 & 2.60 & 21.27 & $<0.0001$ \\
\hline
\end{tabular}




\section{Discussion}

Cancer is particularly complex. Various cancers often have their own risk factors and have unique geographic patterns [13]. The spatial distribution of liver cancer in Longkou City shows that there is obvious spatial heterogeneity, that is, the spatial aggregation and randomness coexist, with many high incidence and high death areas scattered in the distribution, and the whole city exists a number of high incidence "focal points", suggesting a local geographic risk factor. The Moran's $I$ global spatial autocorrelation analysis results of liver cancer showed that the total cumulative incidence of liver cancer has significant spatial correlation, indicating that the incidence of liver cancer in Longkou City has obvious spatial structure, and there may be obvious high incidence areas. This is similar to the reported spatial autocorrelation patterns in the whole of mainland China at the province level [14]. The LISA local spatial autocorrelation analysis results show that, as for the total cumulative incidence, 69 villages are statistically significant, accounting for $10.66 \%$ of the total number of natural villages. The total cumulative mortality rate is statistically significant in 65 villages, accounting for $10.05 \%$ of the total. The spatial scanning and spatio-temporal scanning of liver cancer show that there exist high incidence and high death villages with relatively fixed spatial location in small areas of Longkou City, and the results of time and space scanning and simple spatial scanning are different, indicating that time factors have certain influence on the space area of liver cancer. However, no studies have yet been published revealing the underlying mechanism, thus further studies should be done to explore this finding [15, 16]. This research contributed to the knowledge of the spatial structure and spatial heterogeneity of liver cancer in a small region (village as a unit), and provided a scientific basis for the development of targeted regional prevention and control measures for liver cancer.

\section{Competing Interests}

The authors declare that they have no competing interest.

\section{Authors' Contributions}

JQ extracted the data, conducted the statistical analysis and drafted the manuscript. MBW and FZX conceived of the project concept, helped to interpret the results and modify the manuscript. HYL extracted the data. JX conducted the data analyses. JJX helped write the manuscript. All authors read and approved the final manuscript.

\section{Acknowledgements}

The authors would like would like to thank the tutors for their help conducting the study, notably Professor Maobo Wang and Dr Fuzhong Xue. We would also like to thank Yantai Center for Disease Control and Prevention for providing the liver cancer surveillance data for Longkou City.

\section{References}

[1] Doll R (1967). Prevention of Cancer: Pointers from Epidemiology [M]. London: Nuffeld Provincial Hospitals Trust.

[2] Bing Li, Junyao Li. Death and distribution characteristics of malignant tumors in China [J]. Chinese Journal of oncology, 1980, 2 (1): 1.

[3] SinoMaps Press. The Editoarial Committee for the Atlas of Cancer Mortality in the People's Republic of China. Atlas of cancer mortality in the People's Republic of China [M]. Beijing, China, 1979: 40-46.

[4] Wanqing Chen, Xiaonong Zou, Siwei Zhang. Geographical distribution of mortality of liver cancer in China [J]. Journal of Practical Oncology, 2008, 22 (9): 201-203.

[5] Min Weng, Wanyi Li, Shiliang Su, Zhongliang Cai. Study on the spatial pattern of the incidence of liver cancer from the perspective of social deprivation -- Taking Shenzhen as an example of $[\mathrm{J}]$. Modern preventive medicine, 2016, 43 (8): $1355-1358$.

[6] Zhang W, Wang L, Fang L, et al. Spatial analysis of malaria in Anhui province, China [J]. Malar J, 2008, 7: 206.

[7] Ruihong Liu. Spatial epidemiological characteristics and risk factors of stroke in small areas [D]. Jinan: Shandong University, 2016.

[8] MKF. A spatial scan statistic [J]. Communications in Statistics: Theory and Methods, 1997, 26: 1481-1496.

[9] Luc A. Local indicators of spatial association-LISA [Z]. 1995: 27, 93-115.

[10] Areias C, Briz T, Nunes C. Pulmonary tuberculosis space-time clustering and spatial variation in temporal trends in Portugal, 2000-2010: an updated analysis [J]. Epidemiol Infect, 2015, 143 (15): 3211-3219.

[11] Bellec S, Hemon D, Rudant J, et al. Spatial and space-time clustering of childhood acute leukaemia in France from 1990 to 2000: a nationwide study [J]. Br J Cancer, 2006, 94 (5): 763-770.

[12] Yiannakoulias N. Using population attributable risk to understand geographic disease clusters [J]. Health Place, 2009, 15 (4): 1142-1148.

[13] Wild CP (2012). The role of cancer research in noncommunicable disease control [J]. Natl Cancer Inst, 104: 1051-1058. http://dx.doi.org/10.1093/jnci/djs262.

[14] Aiqiang Xu, Fuzhong Xue, Xiaolei Guo, Jiandong Sun. Status of malignant tumor death and its spatial epidemiology in Shandong Province [M]. Jinan: Shandong Science and Technology Press, 2015 (1): 50.

[15] McGlynn KA, et al (2005). Epidemiology and natural history of hepatocellular carcinoma [J]. Best Practice \& Research in Clinical Gastroenterology, 19 (1): 3-23.

[16] E1-Serag HB. Epidemiology of viral hepatitis and hepatocellular carcinoma [J]. Gastroenterology, 2012, 142 (6): 1264-1273. DOI: 10.1053/j.gastro.2011.12.061. 